\title{
Neural Correlates of Reactivation and Retrieval-Induced Distortion
}

\author{
Donna J. Bridge and Ken A. Paller \\ Interdepartmental Neuroscience Program and Department of Psychology, Northwestern University, Evanston, Illinois 60208
}

Reactivation of recently acquired information can strengthen memory storage and likely contributes to memory consolidation. Retrieval (generating information about prior events) may improve memory storage because it entails reactivation. Alternatively, retrieval may promote storage of retrieved information, and, if retrieval is inaccurate, subsequent recall could be distorted by the retrieved information. If retrieval modifies memory storage, as hypothesized, neural signals associated with accurate retrieval at that time may be distinct from neural signals associated with the degree of repeated retrieval error evident at some later time. We tested this prediction using a 3-session protocol. During session 1, people learned object-location associations to criterion and completed a cued-recall test in which locations were recalled upon viewing objects. During session 2, an electroencephalogram (EEG) was recorded during cued recall for a subset of the associations. During session 3, cued recall was tested for all associations. Retrieval improved storage, in that recall at session 3 was superior for objects tested in session 2 compared with those not tested. Retrieval-induced distortion was revealed in session 3 for those objects tested in session 2, in that those objects were generally placed closer to locations retrieved at session 2 relative to original study locations. EEG analyses revealed positive potentials (400-700 ms) associated with relatively accurate recall at session 2. Memory updating was reflected in positive potentials after $700 \mathrm{~ms}$ that differentially predicted the degree to which recall promoted storage of the session-2-retrieved location. These findings demonstrate unique neurocognitive processing whereby memories are updated with information produced during retrieval.

\section{Introduction}

Memory reactivation refers to neural activity corresponding to information previously learned (Wilson and McNaughton, 1994; O'Neill et al., 2010; Carr et al., 2011). Reactivation of recently acquired events may operate to strengthen associative links among cortical networks that specialize in processing and storing particular types of information. Reactivation could thus facilitate systems consolidation, the gradual process whereby newly acquired memories for facts and events become enduring (Alvarez and Squire, 1994; McClelland et al., 1995; Paller, 2009).

Does retrieval alter memory storage through a reactivationrelated mechanism? Successful retrieval always entails reactivation to some degree, whereas reactivation need not entail conscious retrieval of information about a prior episode. Cueing procedures that engage retrieval produce lasting benefits (Landauer and Bjork, 1978; Karpicke and Roediger, 2008), but the neural mechanisms mediating these benefits are unknown.

Received March 20, 2012; revised June 15, 2012; accepted July 16, 2012.

Author contributions: D.J.B. and K.A.P. designed research; D.J.B. performed research; D.J.B. and K.A.P. analyzed data; D.J.B. and K.A.P. wrote the paper.

This material is based upon work supported by National Science Foundation Grant BCS1025697 and NIH Grant T32 NS047987. We extend gratitude to H. Lucas for providing helpful feedback on the manuscript, to J. Rudoy for designing the spatial association task, and to J. Antony and J. Creery for helpful discussion.

The authors declare no competing financial interests.

Correspondence should be addressed to Donna J. Bridge, Northwestern University, Department of Medical Social Sciences, 710 Lake Shore Drive, Suite 729, Chicago, IL 60611. E-mail: donnajb@u.northwestern.edu.

DOI:10.1523/JNEUROSCI.1378-12.2012

Copyright $\odot 2012$ the authors $\quad 0270-6474 / 12 / 3212144-08 \$ 15.00 / 0$
Retrieval may be effective because it includes reactivation, but it may also enable memory storage to be updated with new information (Dudai and Eisenberg, 2004). During retrieval, environmental information in the current spatiotemporal context could become associated with stored information (Karlsson and Frank, 2009). Also, information retrieved in response to a cue could become integrated into stored memory representations.

Retrieval rarely provides a complete and precise account of prior events; rather, recall often includes both veridical and erroneous information. Therefore, if retrieval promotes storage of retrieved information, memories could come to include information learned during the original event and information activated via erroneous retrieval. This scenario could account for gradual memory distortion, or even mostly false recollection, after multiple recalling and retelling episodes.

Here we examined the degree to which retrieval facilitated veridical memory storage and the degree to which retrieval produced memory distortion in conjunction with errors in retrieval. We hypothesized that retrieval preferentially promotes storage of retrieved information instead of merely strengthening memory for the original event. Retrieval should thus have two distinct consequences. To the extent that correct information is recalled, retrieval will promote subsequent memory accuracy. Conversely, recalled information that diverges from the original event will be incorporated into the memory representation, thereby promoting memory distortion.

We examined the consequences of retrieval by testing objectlocation associations over multiple sessions. Each participant learned to associate objects with unique locations. Then, cuedrecall tests provided fine-grained measures of the degree to which 
participants recalled the correct locations. Results from this test not only provided accuracy indices but also showed the degree to which recall was biased with respect to locations retrieved previously. In this way, we could determine whether retrieval preferentially promoted storage of retrieved information.

As a further test of the hypothesis that an updating mechanism is operative during retrieval, we examined neural signals during cued recall. Prior studies have described specific signals associated with memory formation and retrieval (Friedman and Johnson, 2000; Paller and Wagner, 2002; Rugg and Curran, 2007), but neural correlates of memory change induced by retrieval are uncommon (Buckner et al., 2001). We determined whether unique electroencephalographic (EEG) signals recorded during retrieval predict subsequent memory for retrieved locations.

\section{Materials and Methods}

Participants. Twelve individuals ( 9 women) from the university community participated. Informed consent was given in advance and payment was provided after completion.

Stimuli. A set of 180 color drawings of objects were used (Rossion and Pourtois, 2004). Objects were presented on a background showing a blue-and-red grid. Each object was presented with a central red dot to indicate its precise location on the grid, which could be anywhere such that the whole object was visible on the background. Dot locations thus appeared in a central $600 \times 600$ pixel $(15.63 \times 15.63 \mathrm{~cm})$ region.

Procedure. In session 1, participants learned 180 object-location associations. Each of the 180 objects was associated with a distinct spatial location on the screen. Participants then completed the first of three cued-recall tests (T1), as each object was shown in the center of the screen to serve as a cue to recall the corresponding location. In session 2, a subset of the objects appeared and participants attempted to retrieve associated locations. EEG activity was recorded during this second cued-recall test (T2). In session 3, cued recall was tested for all spatial associations (T3). Each session began $\sim 24 \mathrm{~h}$ after the start of the prior session (mean 23.83 $\mathrm{h}, \mathrm{SE}=0.14$ ).

Testing spatial associations is ideal for providing objective gradations in memory performance. Instead of a binary assessment of memory performance (as in many memory tests) or a subjectively graded response measure (as in recognition with confidence ratings), the distance between the recalled location and the original location provides a graded measure of accuracy. Furthermore, we were also able to evaluate memory on the basis of the extent to which a recalled location was similar to one recalled earlier in response to the same object.

Three experimental conditions were used: Active, Covert, and No Retrieval. In the Active and Covert retrieval conditions, participants were prompted to mentally engage in retrieval during session 2, but they overtly recalled locations only in the Active retrieval condition. We included these two retrieval conditions to determine whether subsequent memory differed as a function of the engagement of overt versus internal retrieval processing. We predicted that recall accuracy during session 3 would be superior for associations retrieved during session 2 than for associations not retrieved during session 2. Because measures of accuracy were obtained at $\mathrm{T} 2$ for the Active but not the Covert retrieval condition, the Active condition was of primary interest. Objects were pseudorandomly assigned to each condition, taking recall accuracy at T1 into account so as to equate initial memory strength across conditions. To provide sufficient signal-to-noise ratios for analyses of electrophysiological correlates of active retrieval processing at T2, 120 objects were assigned to the Active condition, leaving 30 for the Covert condition and 30 for the No Retrieval condition.

In session 1 (Fig. $1 \mathrm{~A}$ ), participants completed 9 learning blocks, each of which included 20 unique object-location associations. This segmented format for learning allowed participants to learn all 180 associations reasonably well, because objects could be repeated at short delays without too much forgetting or interference. At the beginning of every block, a sequence of 20 new objects was presented in a set of random locations for $2000 \mathrm{~ms}$ each. Then, each object was presented in the center of the screen for $500 \mathrm{~ms}$, at which point a sound cue ("click") prompted participants to attempt to move the object to its associated location. Participants dragged the object by operating a computer mouse and clicked the mouse button to indicate their decision. Feedback was provided as the object immediately disappeared from the selected location and reappeared in the correct location. Regardless of response accuracy, the object was shown in this correct location for $2000 \mathrm{~ms}$. After all 20 objects appeared, testing continued with the same set of objects in a different random order. In this way, participants repeatedly practiced retrieval for the same objects via this active recall method to achieve the following learning criterion. A response was considered correct if placed within 150 pixels $(3.9 \mathrm{~cm})$ of the correct location. When an object was placed correctly two consecutive times, it was dropped from the learning rotation. Repetitive list presentation continued until all locations in a block were learned to this criterion. Across participants, the number of practice trials completed per object ranged from 2 to 14, with a mean of 2.99 retrieval trials per object $(\mathrm{SE}=0.2$ ). After the ninth block, a $15 \mathrm{~min}$ break was given, followed by $\mathrm{T} 1$, when participants attempted to recall each object's location using the same procedures as during learning except that each of the 180 objects was presented only once and no feedback was provided. At $\mathrm{T} 1,70 \%$ ( $\mathrm{SE}=4.34 \%$ ) of the objects were recalled within the learning criterion of $3.9 \mathrm{~cm}$, suggesting that some forgetting occurred following the 15 min post-learning delay.

The following day, participants returned to the lab for session 2 (Fig. $1 B)$. EEG was recorded during T2, when objects assigned to Active and Covert conditions appeared in a random order. At the beginning of each trial, a gray screen showed the word "blink," which signaled a rest period and helped to limit blinks and muscle activity during experimental trials. Once ready to proceed, the participant clicked a mouse button and fixated on a cross that appeared in the center of the grid for $1000 \mathrm{~ms}$. Next, objects appeared for spatial-association recall without feedback. For the Active condition, objects were displayed for $1500 \mathrm{~ms}$ before a sound cue was played, prompting participants to move the object to its associated location. For the Covert condition, objects were displayed for $2000 \mathrm{~ms}$ and then disappeared, ending the trial. Given that the $1500 \mathrm{~ms}$ interval at the beginning of each trial was identical in the two conditions, we presume that participants covertly retrieved spatial locations in both conditions, although they actively moved objects to remembered locations only in the Active condition. At T2, 56\% ( $\mathrm{SE}=5.3 \%$ ) of the Active objects were recalled within the learning criterion.

The next day, participants returned for session 3 (Fig. 1C). The final test, T3, was administered using the same procedure used in T1 for all 180 spatial associations. At T3, 53.7\% ( $\mathrm{SE}=4.82 \%)$ of the objects were recalled within the learning criterion.

EEG acquisition and analysis. Continuous electroencephalographic activity was recorded during the session-2 cued-recall test using $\mathrm{Ag} / \mathrm{AgCl}$ active electrodes (BioSemi). Recordings were made from 32 scalp locations (bandwidth DC to $104 \mathrm{~Hz}$, sampling rate $512 \mathrm{~Hz}$ ). Voltage between each electrode site and a common mode sense electrode was measured and amplified during recording; referencing was accomplished offline by averaging the recordings from the left and right mastoid electrodes. Electrooculographic (EOG) recordings were also made using two electrodes lateral to the eyes and two electrodes situated below the eyes to monitor eye movements and blinks. A high-pass filter $(0.1 \mathrm{~Hz}$ cutoff, $12 \mathrm{~dB}$ per octave roll-off) was applied to the data before analysis.

Event-related potentials (ERPs) extracted from the EEG activity were time-locked to the onset of the centrally presented object cues. ERP analysis was conducted on trials in the Active condition only (120 trials). Epochs lasted $1200 \mathrm{~ms}$, beginning $200 \mathrm{~ms}$ before stimulus onset (with baseline correction). Ocular artifacts were detected using a $200 \mathrm{~ms}$ moving window with $100 \mathrm{~ms}$ steps to identify epochs that exhibited large changes in voltage at the EOG channels. An absolute voltage threshold was applied to the remaining channels to detect exceptionally noisy trials due to head movement or muscle tension. Trials with artifacts were removed (26 trials per participant on average), and exceptionally noisy channels were spatially interpolated (1.4 channels per participant on average). Median splits were relied on for ERP analyses, as described below, with an average of 47 trials included for each condition (see Table 
A

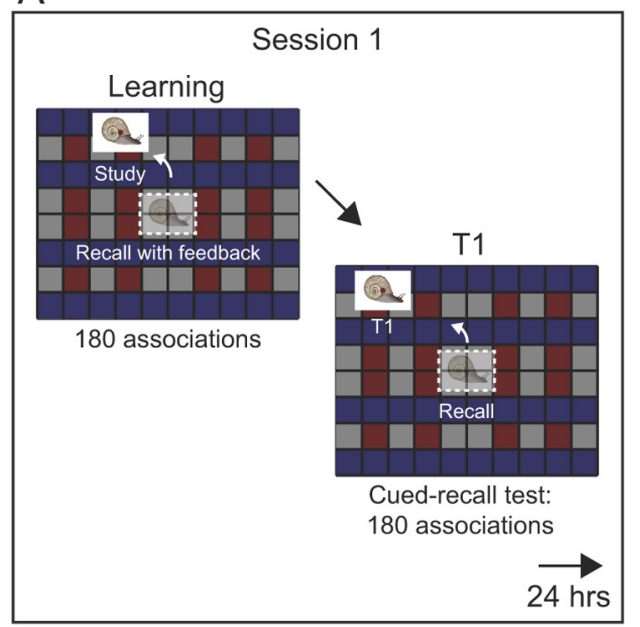

C

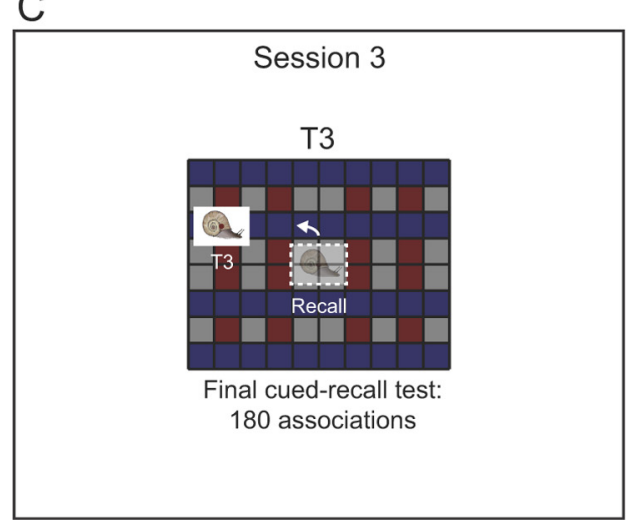

B

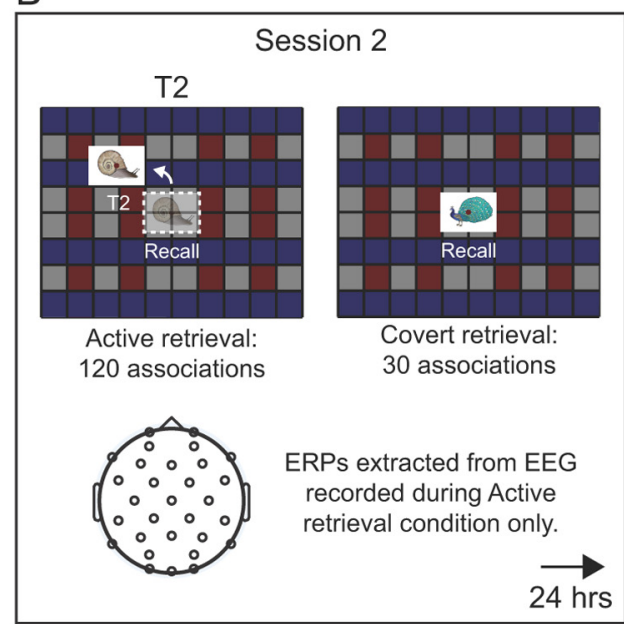

D

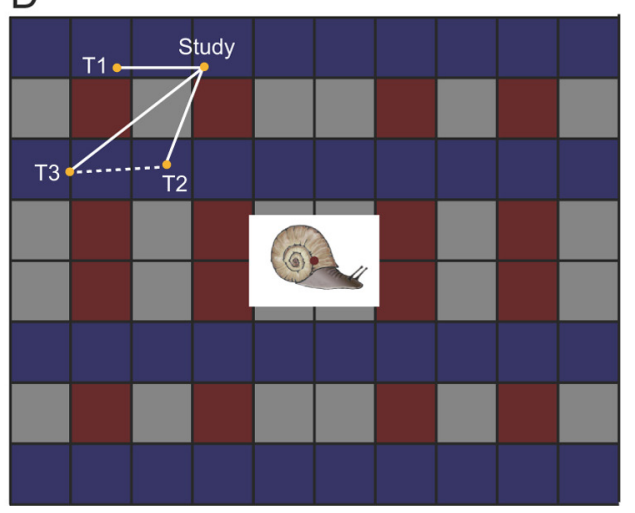

Figure 1. Schematic of the spatial-association task. $A$, At the beginning of the session- 1 learning phase, each object was initially presented in a unique screen location on a $1024 \times 768$ pixel $(26.67 \times 20 \mathrm{~cm})$ grid, viewed from a distance of $92 \mathrm{~cm}$. After all learning trials, participants completed the first cued-recall test (T1). In this test, objects were shown in the center of the screen and participants were prompted to move each object to its original location. B, At session 2, EEG was recorded while participants completed the second cued-recall test (T2). For the Active condition, participants viewed each object for $1500 \mathrm{~ms}$ and then were prompted to move the object to its original location. For the Covert condition, participants prepared to recall the original location as in the Active condition, and likely attempted to retrieve the location during this time, but they had no opportunity to move the object. Trials in the Active and Covert conditions were randomly intermixed. C, At session 3, participants completed the final cued-recall test (T3). D, The original location for one example object in the Active condition is labeled "Study." Solid white lines depict the distance the object was placed from the study location at each test. Typical errors made on each of the three tests as shown: mean error of $3.59 \mathrm{~cm}$ at $\mathrm{T} 1$, mean error of $4.73 \mathrm{~cm}$ at $\mathrm{T2}$ (Active condition only), and mean error of $5.01 \mathrm{~cm}$ at T3. The dashed white line depicts the distance the object was placed from the T2 retrieved location at the final test (retrieval bias distance).

Table 1. Trial sorting conditionalized according to median splits

\begin{tabular}{llll}
\hline & Above median (closer) & Median & Below median (farther) \\
\hline Current accuracy & $1.89(0.27)$ & $3.64(1.05)$ & $7.57(0.62)$ \\
Future accuracy & $2.01(0.24)$ & $3.74(1.08)$ & $7.84(0.59)$ \\
Future retrieval bias & $0.99(0.13)$ & $2.14(0.62)$ & $6.19(0.67)$ \\
\hline
\end{tabular}

For each contrast, trials were distributed into two conditions (Closer/Farther) according to distance measurements. These distance measurements were obtained on the basis of current accuracy, future accuracy, and future retrieval bias. The distance between the $\mathrm{T} 2$ recalled location and the study location was used for current accuracy. The distance between the $T 3$ recalled location and the study location was used for future accuracy. The distance between the $\mathrm{T} 3$ recalled location and the $\mathrm{T} 2$ recalled location was used for future retrieval bias. Trials were assigned to conditions according to whether distances were smaller or greater than the median. Distance values are shown as averaged across participants (measured in centimeters, with SEM).

1 for corresponding behavioral data). A $30 \mathrm{~Hz}$ low-pass filter was applied for waveform presentation.

Although few ERP studies have examined neural signals during retrieval that predict subsequent memory performance, many studies have identified potentials during encoding and retrieval that vary as a function of memory success. ERP differences at encoding that predict subsequent memory performance typically arise $\sim 400 \mathrm{~ms}$ post-cue onset and are prominent at lateral parietal scalp locations (Paller et al., 1987; Paller and Wagner, 2002). ERPs corresponding to successful recognition of previously studied material tend to exhibit late-positive deflections at both frontal and parietal locations (Friedman and Johnson, 2000; Van Petten et al., 2000; Rugg and Curran, 2007). Accordingly, average amplitudes were computed for two $300 \mathrm{~ms}$ time intervals that spanned the mid (400-700 ms) and late (700-1000 ms) phases of the epoch. Regional effects were assessed by collapsing data from eight parietal electrodes (T7, T8, CP5 , CP6, P3, P4, P7, and P8) and eight frontal electrodes (FP1, FP2, AF3, AF4, F3, F4, F7, and F8). In reporting ANOVA results, we focus on effects with relevance to memory performance (omitting main effects of time or region). Error probability was adjusted using the Huynh/Feldt correction to account for violations of sphericity (denoted ${ }_{\mathrm{HF}}$ when applied to analyses in text).

\section{Results}

\section{Behavioral data}

Memory accuracy

To determine whether there was a memory benefit from retrieval engaged during session 2, we analyzed recall accuracy for each experimental condition by calculating mean error scores (distance between recalled location and original location, as shown in Fig. 1D). After T1, but before the session-2 manipulation, trials were assigned to conditions such that mean accuracy was matched across the three conditions for each participant. A oneway ANOVA confirmed that the mean error at T1 did not differ across conditions $\left(F_{(1.34,14.73)}=0.397\right.$, n.s. .HF $)$. 


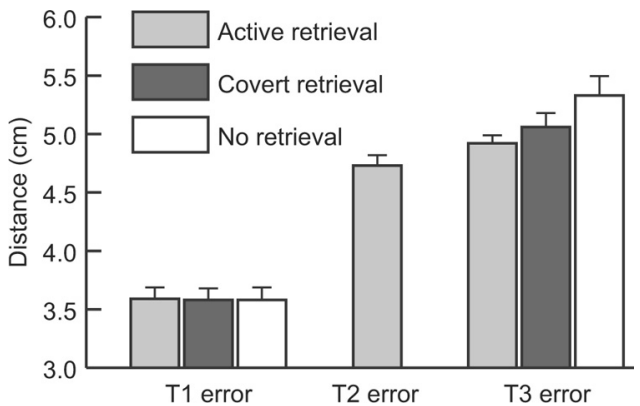

Figure 2. Mean errors in the spatial-association task. Errors on each test (T1, $\mathrm{T} 2$, and $\mathrm{T} 3$ ) were computed as the distance objects were placed from corresponding study locations. At T3, forgetting across days was significantly less for objects in the Active Retrieval condition versus the No Retrieval condition. Over all three conditions, errors were greater on T3 than on T1 ( $t_{(11)}$ $=8.77, p<0.001)$. For the Active Retrieval condition, errors were greater on T2 than on T1 $\left(t_{(11)}=5.07, p<0.001\right)$ and greater on $\mathrm{T} 3$ than on $\mathrm{T} 2\left(t_{(11)}=2.42, p<0.05\right)$. Error bars show SEM after removing across-subject variability.

Given no initial disparities in recall accuracy, we assessed mean errors at T3 for each condition (Fig. 2). As expected, active retrieval at $\mathrm{T} 2$ led to an improvement in recall accuracy at $\mathrm{T} 3$ compared with no retrieval at T2 $\left(t_{(11)}=2.94, p<0.05\right)$. The Covert condition might be expected to include a subset of the processing in the Active condition, and thus also be somewhat beneficial. Indeed, a linear trend was found such that $\mathrm{T} 3$ recall was greatest for the Active condition, intermediate for the Covert condition, and lowest for the No Retrieval condition. $\left(F_{(1,11)}=\right.$ 8.63, $p<0.05)$. However, accuracy in the Covert condition did not differ reliably between either of the other two conditions

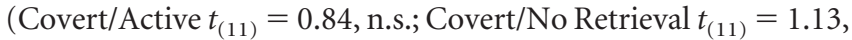
n.s.). Additional analyses of forgetting across days (subtracting T1 error scores from T3 error scores) confirmed the same patterns of condition effects (as expected given no differential effects at T1).

\section{Retrieval bias}

A central goal was to test the hypothesis that retrieval preferentially promotes storage of retrieved information. Because recall at T2 diverged from the original study location on every trial, we were able to use these fine-grained recall measures to determine the extent to which final recall conformed to the T2 retrieved locations. We reasoned that the degree to which objects were placed closer to the T2 locations than to the original study locations was indicative of retrieval-induced distortion. Therefore, we examined final recall performance for the Active condition on the basis of the distance objects were placed from the T2 location for that same object (retrieval bias) and compared these distances to the T3 errors (memory accuracy). Overall, objects were placed significantly closer to T2 locations than to study locations $\left(t_{(11)}=\right.$ 8.26, $p<0.001)$. Analyses of the distribution of responses as a function of distance from the study and T2 locations (Fig. 3) indicated that this effect was driven by trials with the smallest distances. More than $50 \%$ of Active objects were placed within 2 $\mathrm{cm}$ of the T2 location, whereas $\sim 30 \%$ were placed within $2 \mathrm{~cm}$ of the study location. Thus, less-than-perfect recall at T2 produced retrieval-induced distortion on the final test, in that recall was biased in the direction of the $\mathrm{T} 2$ retrieved locations.

To further interrogate the extent to which retrieval at T2 produced distortion on the final test, we subtracted the retrieval bias distances from the memory accuracy distances to obtain a distortion index for each trial in the Active condition. A distortion index $>0$ indicates that the object was placed closer to the T2

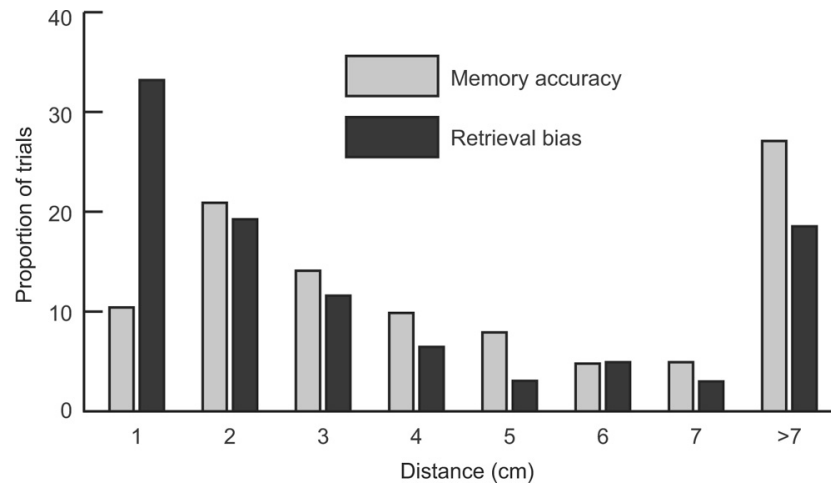

Figure 3. Retrieval bias contrasted with memory accuracy for the Active condition. Locations recalled at $\mathrm{T} 3$ were $4.92 \mathrm{~cm}(\mathrm{SE}=0.06)$ from the study location on average (memory accuracy) and $3.59 \mathrm{~cm}$ (SE $=0.06$ ) from the $T 2$ retrieved location on average (retrieval bias). Bins correspond to the proportion of objects placed fewer than the indicated number of centimeters from the study location or from the $T 2$ retrieved location. Recalled locations were within $1 \mathrm{~cm}$ of the $\mathrm{T} 2$ retrieved location much more often then they were within $1 \mathrm{~cm}$ of the study location.

retrieved location than to the study location, whereas a distortion index $<0$ indicates that the object was placed closer to the study location than to the T2 retrieved location. On average, $69 \%$ of the objects in the Active condition had distortion indices $>0$, indicating that the majority of objects were placed closer to the corresponding $\mathrm{T} 2$ retrieved location than to the study location.

Although distortion on the final test was evident for the majority of objects in the Active condition, one possible scenario is that retrieval-induced distortion occurred primarily on trials when the original location had been forgotten at T2. To systematically determine whether poor accuracy at T2 was a requirement for retrieval-induced distortion, we compared T2 accuracy for objects with a distortion index $>0$ to T2 accuracy for objects with a distortion index $<0$. Interestingly, retrieval-induced distortion on the final test did not depend on T2 accuracy; the mean $\mathrm{T} 2$ error was $4.68 \mathrm{~cm}(\mathrm{SE}=0.42)$ for objects with retrievalinduced distortion and $4.90 \mathrm{~cm}(\mathrm{SE}=0.53)$ for objects without retrieval-induced distortion, a nonsignificant difference $\left(t_{(11)}=\right.$ 0.49 , n.s.). Thus, the level of accuracy at T2 was nearly the same on trials when distortion was evident versus trials when distortion was not evident, further supporting the notion that retrieval promotes memory for the retrieved information.

\section{Electrophysiology}

The Active condition provided an opportunity to interrogate neural signals produced during retrieval at session 2. Relevant neural activity can be contrasted both as a function of current memory performance and as a function of later memory performance. The former contrast should reveal processing correlated with memory retrieval at that moment during $\mathrm{T} 2$, whereas the latter contrast may reveal processing that in part determines what will be remembered the next day. ERPs at T2 for the Active condition were thus subjected to analyses as a function of current accuracy, future accuracy, and future retrieval bias. The two future memory contrasts are akin to subsequent memory analyses, in which ERPs at encoding are examined as a function of subsequent memory (Paller and Wagner, 2002), here yielding ERP differences termed Dm for future accuracy and Dm for future retrieval bias.

The behavioral data used to conduct median-split ERP analyses are summarized in Table 1. In the analysis as a function of current accuracy, ERPs were computed using a median split of 
the T2 errors. We thus compared T2 ERPs for objects placed closer to the original location versus those placed farther from the original location at T2, with closer distances reflecting high accuracy and farther distances reflecting low accuracy. In the analysis as a function of future accuracy, ERPs were computed using a median split of the T3 errors. We thus compared T2 ERPs for objects placed closer to the original location at the next session (high accuracy) to ERPs for objects placed farther from the original location at the next session (low accuracy). In the analysis as a function of future retrieval bias, T2 ERPs were computed using a median split of the retrieval bias distances, which are based on T2 locations rather than original locations. In other words, we compared objects placed closer to the T2 location at the next session to those placed farther from the T2 location at the next session. Based on these measures, we describe closer distances as reflecting high retrieval bias and farther distances as reflecting low retrieval bias.

With these procedures, we used the same trials to examine ERPs on the basis of current accuracy, future accuracy, and future retrieval bias; trials were segregated differently in the three analyses such that the analyses revealed different results. For the current and future accuracy contrasts, $23 \%$ of the trials were assigned to different accuracy bins (i.e., high current accuracy and low future accuracy or the reverse). For the current accuracy and future retrieval bias contrasts, $37 \%$ of the trials were assigned to different bins (i.e., high current accuracy and low future retrieval bias or the reverse). For the future accuracy and future retrieval bias contrasts, $38 \%$ of the trials were assigned to different bins (i.e., high future accuracy and low future retrieval bias or the reverse). Although these trials with different assignments were key to obtaining different ERP results in the three contrasts, it was necessary to use all trials to have sufficient signal-tonoise ratios for ERP measurements.

We conducted a series of analyses separately for each memory contrast. Beginning at $400 \mathrm{~ms}$, mean amplitudes computed over $300 \mathrm{~ms}$ intervals were subjected to separate repeated-measures ANOVAs with distance (close, far) and region (frontal, parietal) as independent variables.

\section{Current accuracy}

In line with studies that have examined ERPs as a function of successful retrieval (Paller, 2004; Rugg and Curran, 2007), we predicted that current accuracy ERPs would show late positive differences at frontal and parietal sites. If neural reactivation occurs during retrieval (Nyberg et al., 2000; Wheeler et al., 2000; Jacobs et al., 2012), ERPs corresponding to performance differences in current accuracy could reflect reactivation of the original association.

Visual inspection of the current accuracy waveforms indicated prominent differences in latter portions of the recording epoch at frontal and parietal regions (Fig. 4A). Indeed, ERPs at 400-700 ms appeared to be more positive for objects recalled at T2 with high accuracy relative to low accuracy. Statistical analysis confirmed that amplitudes were significantly greater for objects placed closer to the original locations relative to those placed farther from the original locations $\left(F_{(1,11)}=8.41, p<0.05\right)$. This effect of distance was similar at frontal and parietal sites $\left(F_{(1,11)}=\right.$ 1.84 , n.s.). This effect appeared to decline late in the epoch. At 700-1000 ms, neither the main effect of distance nor the interaction with region were significant $\left(F_{(1,11)}=1.63\right.$, n.s.; $F_{(1,11)}=$ 0.06 , n.s.).

Future accuracy

To the extent that retrieval promotes memory for the original association, neural signals at retrieval might differentiate on the basis of subsequent memory for the original study location. Based on visual inspection of the ERPs corresponding to future accuracy, negligible differences were evident during either time inter$\operatorname{val}($ Fig. $4 B$ ). Average amplitudes computed on the basis of future accuracy did not reveal significant differences during any time interval. ERPs corresponding to objects placed closer to the original locations versus those placed farther from the original locations at T3 did not differ at $400-700 \mathrm{~ms}\left(F_{(1,11)}=0.00\right.$, n.s. $)$ nor $700-1000 \mathrm{~ms}\left(F_{(1,11)}=0.08\right.$, n.s. $)$. No interactions with region were observed $(F$ values $<0.6)$.

\section{Future retrieval bias}

One possible explanation for the lack of ERP differences at session 2 as a function of future accuracy is that neural processing at T2 was not simply devoted to the maintenance of memory for the original object-location association. Processing may have been dominated by the retrieved location, which always diverged from the original location. Accordingly, we hypothesized that ERPs at $\mathrm{T} 2$ would uniquely predict the extent to which $\mathrm{T} 3$ recall recapitulated $\mathrm{T} 2$ retrieved locations.

Visual inspection of ERPs sorted on the basis of future retrieval bias (Fig. $4 C$ ) indicated differences that emerged at $\sim 500$ $\mathrm{ms}$ at parietal locations, later at frontal locations, and persisted until the end of the epoch. ANOVA results indicated that amplitudes at 400-700 ms did not differ on the basis of future retrieval bias $\left(F_{(1,11)}=2.05\right.$, n.s. $)$, and the interaction with region was not significant $\left(F_{(1,11)}=2.99\right.$, n.s. $)$. Amplitudes at 700-1000 ms were greater for objects placed closer to $\mathrm{T} 2$ retrieved locations relative to objects placed farther from $\mathrm{T} 2$ retrieved locations $\left(F_{(1,11)}=\right.$ $7.86, p<0.05)$. During the late time interval, this enhanced positivity was similarly evident at frontal and parietal locations $\left(F_{(1,11)}=0.08\right.$, n.s. $)$.

Spatial topography: Current accuracy versus future retrieval bias We hypothesized that distinct mechanisms mediated the current accuracy and future retrieval bias effects. To directly compare these effects topographically, we first calculated difference waves (closer minus farther) for both contrasts (Fig. 5). Difference amplitudes were then normalized according to the procedure recommended by McCarthy and Wood (1985). The comparison of the normalized difference amplitudes at 400-700 $\mathrm{ms}$ in a repeated-measures ANOVA with memory contrast and location (32 scalp electrodes) as factors revealed a significant interaction of memory contrast and location $\left(F_{(16.5,181.47)}=2.12, p<\right.$ $\left.0.01_{\mathrm{HF}}\right)$. Whereas the prior analyses for the interval from 400 to $700 \mathrm{~ms}$ showed significant amplitude differences on the basis of current accuracy but not on the basis of future retrieval bias, these results indicate that the spatial topography of these two memory contrasts were also distinct.

Next, we examined whether the two effects differed topographically in the late time interval, when amplitude differences were only observed on the basis of future retrieval bias. At $700-$ $1000 \mathrm{~ms}$, topographical differences across the contrasts were nonsignificant $\left(F_{(9.81,107.9)}=0.81\right.$, n.s. $\left.{ }_{\mathrm{HF}}\right)$.

Finally, we aimed to determine if the contrasts differed topographically during the time intervals with reliable amplitude effects. We compared the effect based on current accuracy at $400-700 \mathrm{~ms}$ with the effect based on future retrieval bias at $700-$ $1000 \mathrm{~ms})$. We observed a nonsignificant interaction of memory contrast and location $\left(F_{(15.49,170.39)}=1.6, p<0.077_{\mathrm{HF}}\right)$.

\section{Discussion}

Neural correlates of retrieval-induced distortion have never been examined previously. Many studies of how retrieval influences 

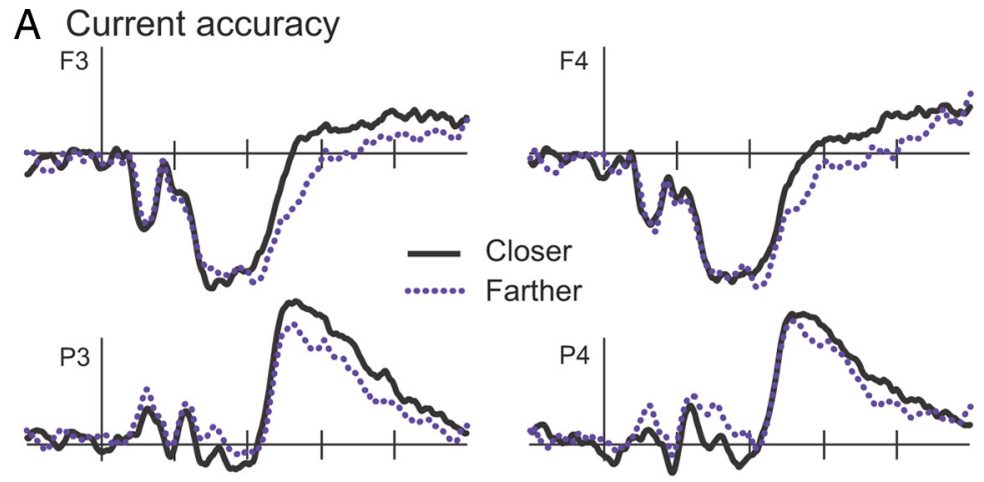

B Future accuracy
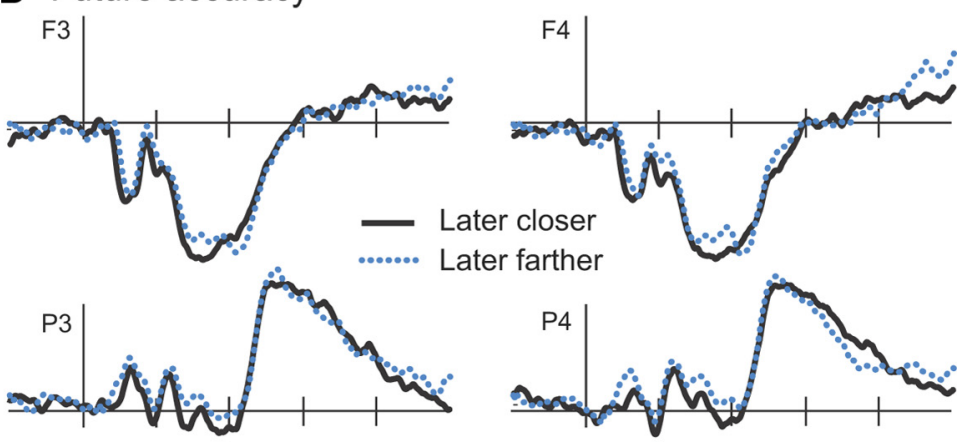

C Future retrieval bias
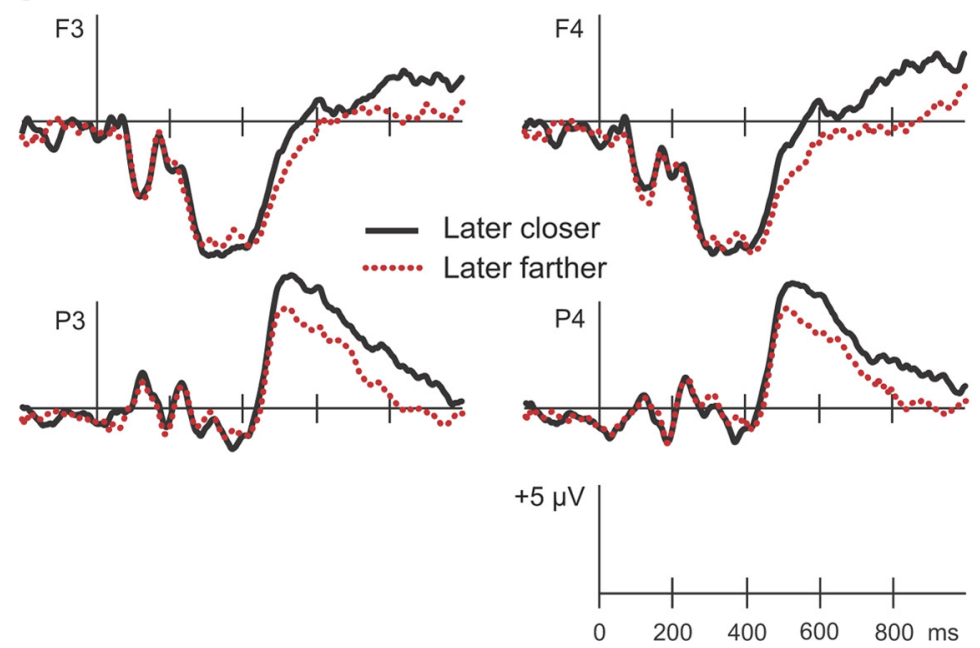

Figure 4. Electrophysiological results. ERPs recorded at session 2 were computed for objects in the Active condition placed closer to (solid lines) or farther from (dotted lines) the corresponding study or T2 locations. $\boldsymbol{A}$, Current accuracy reflects distance objects were placed from the original location at T2. $\boldsymbol{B}$, Future accuracy reflects distance objects were placed from the original location at T3. C, Future retrieval bias reflects distance objects were placed from the T2 retrieved location at T3. ERPs from two frontal (F3 and F4) and two parietal (P3 and P4) locations are displayed.

subsequent memory have focused on the "testing effect" (Landauer and Bjork, 1978; Thompson et al., 1978; Carrier and Pashler, 1992; Wheeler and Roediger, 1992; Karpicke and Roediger, 2008; Roediger and Butler, 2011), which refers to the finding that recalling an associate in response to a cue during learning enhances memory storage compared with simply studying a cue-associate pair. Apparently, the testing effect is not completely beneficial. For instance, lures incorrectly selected on an initial multiple-choice test were frequently recalled on subsequent tests (Roediger and Marsh, 2005; Butler et al., 2006; Marsh et al., 2007), suggesting that erroneously retrieved information interferes with future memory performance. Here, recall on $\mathrm{T} 3$ was typically off target in the direction of the T2 location, which was not identical to the original location. Accord- ingly, retrieval did not necessarily reinforce the original association; rather, it altered storage by reinforcing the association that was retrieved on $\mathrm{T} 2$.

Indeed, beneficial effects of retrieval on memory may be mediated by (1) reactivation of the original study location and/or (2) storage of the retrieved location, which tends to be related to (but not necessarily identical to) the original location. Although reactivation has been shown to facilitate subsequent memory performance (Rasch et al., 2007; Rudoy et al., 2009; Dupret et al., 2010; Xue et al., 2010), the possibility that retrieval has more complex effects is supported by the finding that brain potentials in the present study corresponding to current accuracy were distinct from those that predicted future retrieval bias. We propose that future recall was not solely dependent upon successful retrieval of the original location; rather, future recall was noticeably influenced by plasticity related to the $\mathrm{T} 2$ retrieved location.

As suggested by the retrieval-induced distortion results observed here, retrieval promoted encoding and storage of the retrieval event itself. During retrieval, neural plasticity is enhanced in the CA1 region of the hippocampus (Dupret et al., 2010), which could promote the integration of new information in the context of preexisting memory representations. Indeed, other studies have also shown that encoding processes are operative during retrieval tests (Buckner et al., 2001; Dudukovic et al., 2009). For instance, lures on a recognition test were remembered subsequently on an unexpected memory test, with accuracy comparable to that of items intentionally encoded during a traditional study task (Buckner et al., 2001). These findings fit with the notion that the T2 retrieved location was encoded at the time of testing and subsequently remembered on the final test. However, we cannot rule out the possibility that memory for the original location deteriorated before retrieval at T2, and that retrieval at session 2 provided a rehearsal opportunity for that erroneous location. Regardless of whether the location recalled at T2 was generated just at that moment, the electrophysiological retrieval bias effect signifies processing of the erroneous location that was operative in determining later recall performance. The behavioral results from the final test show unequivocally that recall was biased in the direction of retrieval at $\mathrm{T} 2$.

Although performance measures in this spatial memory task were subject to retrieval-induced distortion, retrieval both improved and distorted memory. At session 2, participants revisited the object-location associations they learned the prior day, and this engagement of retrieval facilitated memory accuracy at session 3 (Fig. 2). At the same time, distortion was introduced by retrieval, in that locations recalled at session 3 were biased in the 
A Current accuracy
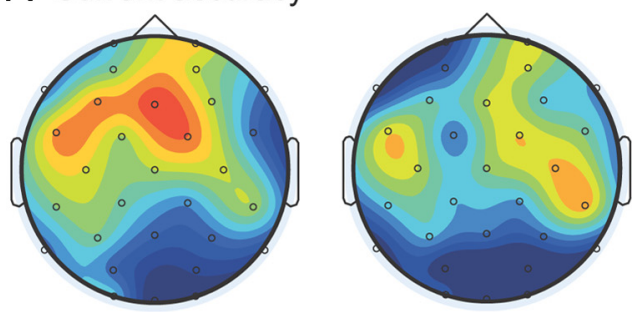

B Future accuracy
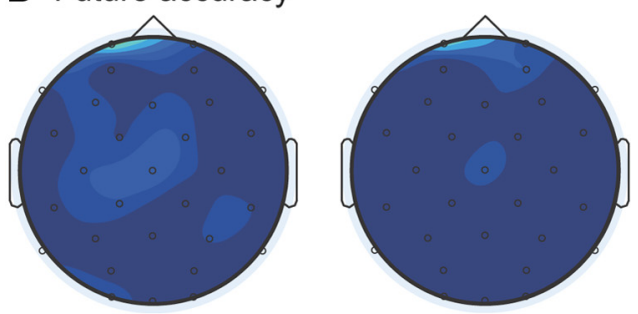

C Future retrieval bias

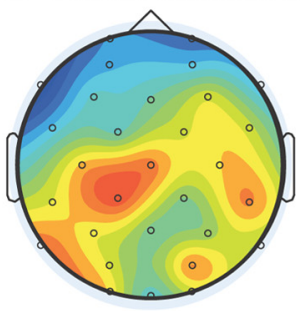

400-700 ms

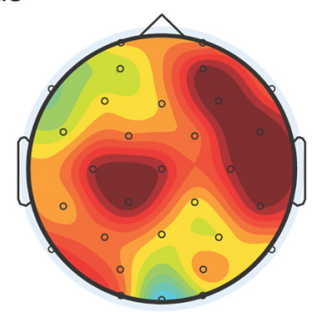

$700-1000 \mathrm{~ms}$

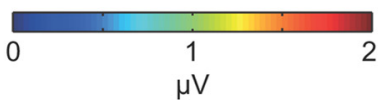

Figure 5. Topographic maps. $\boldsymbol{A}-\boldsymbol{C}$, Individual maps depict mean amplitude differences at $400-700 \mathrm{~ms}$ and $700-1000 \mathrm{~ms}$ for the contrast current accuracy $(\boldsymbol{A})$, the contrast future accu$\operatorname{racy}(\boldsymbol{B})$, and the contrast future retrieval bias $(\boldsymbol{C})$. Whereas differences tended to be relatively larger at frontal locations for the current accuracy contrast at 400-700 ms, differences were pronounced at most scalp locations at $700-1000 \mathrm{~ms}$ for the future retrieval bias contrast.

direction of locations retrieved at session 2 (Fig. 3). Fine-grained measures obtained for each spatial-association recall response provided high sensitivity that revealed this retrieval-induced distortion. Retrieval-induced distortion may be difficult to observe in memory tests that do not provide such fine-grained measures of recall, but it may nevertheless occur widely following intervening retrieval events.

EEG recordings during retrieval provided a novel perspective. Our analysis strategy was to segregate the same set of trials in multiple ways to reveal three different memory relationships. These relationships were thus compared without confounding factors that would have been present if analyses were instead conducted across different participants or different task conditions. Brain activity that predicted recall accuracy at the time of retrieval diverged from brain activity that predicted recall performance the next day. This distinction provides the first isolation of neural activity at retrieval that reflects the incorporation of new-and often erroneous_-information into a memory. ERPs predicted not absolute accuracy but the degree to which recall on T3 was biased in the direction of the T2 location. This unique brain activity predicting next-day performance thus constitutes a neural correlate of alterations in memory storage produced by retrieval.
Van Petten et al. (2000) also examined recognition of objectlocation associations. Participants were required to indicate whether a specific spatial location was that of a specific object viewed earlier. ERP differences similar to those reported here were found, in both cases probably reflecting reconstruction of the prior event. In general, source memory may entail reconstruction of the prior event, including spatial information that is not always precisely correct. Here, recall errors in the final test revealed the consequences of erroneous retrieval for subsequent memory.

Because neural reactivation is generally believed to occur whenever successful retrieval occurs, we infer that ERPs at T2 reflect both attempted reactivation as well as processing of incorrect locations. Neural reinstatement of prior events can occur during successful retrieval (Nyberg et al., 2000; Wheeler et al., 2000; Gelbard-Sagiv et al., 2008; Dupret et al., 2010; Jacobs et al., 2012). For instance, human hippocampal neuronal activity evoked by specific encoding events reemerges before successful recall (Gelbard-Sagiv et al., 2008). Interestingly, reinstatement of hippocampal network activity during retrieval also predicts subsequent memory in animals. After rats learned spatial associations, synchronous firing of CA1 place cells was enhanced when the animal visited reward locations, and this activity correlated with subsequent memory performance (Dupret et al., 2010).

The same mechanism that promotes subsequent memory for errors generated during retrieval may also promote memory for information that is accurately rehearsed during retrieval. If a memory is comprised of several distinct components tied together via hippocampal-based associations such as the unique spatial and temporal context, multisensory information, and emotional content (Bunsey and Eichenbaum, 1996; Eichenbaum et al., 1996), only those aspects that are reinstated during retrieval will be strengthened. For those components that are inaccurately recalled, memory distortion can arise. Therefore, episodic memories may be comprised of a combination of accurate information that was reinforced through retrieval and erroneous information also processed during retrieval. In this way, intervening instances of memory retrieval may be integral to the consolidation process, whereby new information is rapidly learned and integrated with existing representations.

At the final test, object-location associations retrieved at T2 were more accessible than were the originally studied associations. Differences in recall accessibility may have occurred because retrieval modified memory for the original associations, or because it led to the formation of new spatial associations that were remembered on the final test. Future studies should investigate the extent to which neural representations of objectlocation associations in the hippocampus change with repeated testing. Here we were unable to compare the precise neuroanatomical characteristics of specific memories at encoding and subsequent retrieval events. Future applications of neuroimaging techniques may be useful for determining whether a patternseparation mechanism is operative during retrieval, such that each retrieval event is represented by a distinct pattern of activity in the hippocampus, or whether retrieval induces a patterncompletion process, whereby original object-location representations are modified to incorporate information generated during retrieval (Yassa and Stark, 2011).

The effect of retrieval-induced distortion is reminiscent of reconsolidation, when remote memories are apparently rendered temporarily labile and susceptible to interference following exposure to a reminder cue. Such changes in memory storage can occur when the hippocampus is no longer required for retrieval, 
potentially producing memory disruption (Debiec et al., 2002; Nader, 2003) or temporary inaccessibility (Riccio et al., 2006). Unlike studies wherein retrieval was manipulated during the learning phase (Landauer and Bjork, 1978; Thompson et al., 1978; Carrier and Pashler, 1992; Karpicke and Roediger, 2008), here we examined the influence of a delayed retrieval event on subsequent memory for spatial information. Because a $24 \mathrm{~h}$ delay followed the encoding session, we can speculate that some consolidation processes took place before the retrieval manipulation at session 2 that promoted the stabilization of the memory for the original location. Accordingly, we showed that individual memories were modified by interference produced at T2, leading to retrieval-induced distortion on $\mathrm{T} 3$. We cannot determine whether memory storage was temporarily destabilized during reactivation at session 2, or whether this was merely an opportune time for modification of memory storage in the form of the incorporation of the $\mathrm{T} 2$ retrieved location. In either case, this updating mechanism that occurs to memories during retrieval may be a natural component of consolidation that is essential for strengthening some aspects of memory storage while also facilitating the integration of new information.

\section{References}

Alvarez P, Squire LR (1994) Memory consolidation and the medial temporal lobe: a simple network model. Proc Natl Acad Sci U S A 91:7041-7045.

Buckner RL, Wheeler ME, Sheridan MA (2001) Encoding processes during retrieval tasks. J Cogn Neurosci 13:406-415.

Bunsey M, Eichenbaum H (1996) Conservation of hippocampal memory function in rats and humans. Nature 379:255-257.

Butler AC, Marsh EJ, Goode MK, Roediger HL III (2006) When additional multiple-choice lures aid versus hinder later memory. Appl Cogn Psychol 20:941-956.

Carr MF, Jadhav SP, Frank LM (2011) Hippocampal replay in the awake state: a potential substrate for memory consolidation and retrieval. Nat Neurosci 14:147-153.

Carrier M, Pashler H (1992) The influence of retrieval on retention. Mem Cognit 20:633-642.

Debiec J, LeDoux JE, Nader K (2002) Cellular and systems reconsolidation in the hippocampus. Neuron 36:527-538.

Dudai Y, Eisenberg M (2004) Rites of passage of the engram: reconsolidation and the lingering consolidation hypothesis. Neuron 44:93-100.

Dudukovic NM, Dubrow S, Wagner AD (2009) Attention during memory retrieval enhances future remembering. Mem Cognit 37:953-961.

Dupret D, O’Neill J, Pleydell-Bouverie B, Csicsvari J (2010) The reorganization and reactivation of hippocampal maps predict spatial memory performance. Nat Neurosci 13:995-1002.

Eichenbaum H, Schoenbaum G, Young B, Bunsey M (1996) Functional organization of the hippocampal memory system. Proc Natl Acad Sci U S A 93:13500-13507.

Friedman D, Johnson R Jr (2000) Event-related potential (ERP) studies of memory encoding and retrieval: a selective review. Microsc Res Tech $51: 6-28$.

Gelbard-Sagiv H, Mukamel R, Harel M, Malach R, Fried I (2008) Internally generated reactivation of single neurons in human hippocampus during free recall. Science 322:96-101.

Jacobs J, Lega B, Anderson C (2012) Explaining how brain stimulation can evoke memories. J Cogn Neurosci 24:553-563.

Karlsson MP, Frank LM (2009) Awake replay of remote experiences in the hippocampus. Nat Neurosci 12:913-918.
Karpicke JD, Roediger HL 3rd (2008) The critical importance of retrieval for learning. Science 319:966-968.

Landauer TK, Bjork RA (1978) Optimum rehearsal patterns and name learning. In: Practical aspects of memory (Gruneberg MM, Morris PE, Sykes RN, eds), pp 625-632. London: Academic.

Marsh EJ, Roediger HL 3rd, Bjork RA, Bjork EL (2007) The memorial consequences of multiple-choice testing. Psychon Bull Rev 14:194-199.

McCarthy G, Wood CC (1985) Scalp distributions of event-related potentials: an ambiguity associated with analysis of variance models. Electroencephalogr Clin Neurophysiol 62:203-208.

McClelland JL, McNaughton BL, O'Reilly RC (1995) Why there are complementary learning systems in the hippocampus and neocortex: insights from the successes and failures of connectionist models of learning and memory. Psychol Rev 102:419-457.

Nader K (2003) Memory traces unbound. Trends Neurosci 26:65-72.

Nyberg L, Habib R, McIntosh AR, Tulving E (2000) Reactivation of encoding-related brain activity during memory retrieval. Proc Natl Acad Sci U S A 97:11120-11124.

O’Neill J, Pleydell-Bouverie B, Dupret D, Csicsvari J (2010) Play it again: reactivation of waking experience and memory. Trends Neurosci $33: 220-229$.

Paller KA (2004) Electrical signals of memory and the awareness of remembering. Curr Dir Psychol Sci 13:49-55.

Paller KA (2009) Memory consolidation: systems. In: Encyclopedia of neuroscience (Squire LR, ed) pp 741-749. Oxford: Academic.

Paller KA, Wagner AD (2002) Observing the transformation of experience into memory. Trends Cogn Sci 6:93-102.

Paller KA, Kutas M, Mayes AR (1987) Neural correlates of encoding in an incidental learning paradigm. Electroencephalogr Clin Neurophysiol 67:360-371.

Rasch B, Büchel C, Gais S, Born J (2007) Odor cues during slow-wave sleep prompt declarative memory consolidation. Science 315:1426-1429.

Riccio DC, Millin PM, Bogart AR (2006) Reconsolidation: a brief history a retrieval view, and some recent issues. Learn Mem 13:536-544.

Roediger HL 3rd, Butler AC (2011) The critical role of retrieval practice in long-term retention. Trends Cogn Sci 15:20-27.

Roediger HL 3rd, Marsh EJ (2005) The positive and negative consequences of multiple-choice testing. J Exp Psychol Learn Mem Cogn 31:1155-1159.

Rossion B, Pourtois G (2004) Revisiting Snodgrass and Vanderwart's object set: the role of surface detail in basic-level object recognition. Perception 33:217-236.

Rudoy JD, Voss JL, Westerberg CE, Paller KA (2009) Strengthening individual memories by reactivating them during sleep. Science 326:1079.

Rugg MD, Curran T (2007) Event-related potentials and recognition memory. Trends Cogn Sci 11:251-257.

Thompson CP, Wenger SK, Bartling CA (1978) How recall facilitates subsequent recall: a reappraisal. J Exp Psychol Hum Learn 4:210-221.

Van Petten C, Senkfor AJ, Newberg WM (2000) Memory for drawings in locations: spatial source memory and event-related potentials. Psychophysiology 37:551-564.

Wheeler MA, Roediger HL III (1992) Disparate effects of repeated testing: Reconciling Ballard's (1913) and Bartlett's (1932) results. Psychol Sci 3:240-245

Wheeler ME, Petersen SE, Buckner RL (2000) Memory's echo: vivid remembering reactivates sensory-specific cortex. Proc Natl Acad Sci U S A 97:11125-11129.

Wilson MA, McNaughton BL (1994) Reactivation of hippocampal ensemble memories during sleep. Science 265:676-679.

Xue G, Dong Q, Chen C, Lu Z, Mumford JA, Poldrack RA (2010) Greater neural pattern similarity across repetitions is associated with better memory. Science 330:97-101.

Yassa MA, Stark CE (2011) Pattern separation in the hippocampus. Trends Neurosci 34:515-525. 